\title{
Effectiveness of Community Integrated Intermediary Care (CIIC) Service Model to Enhance Family-Based Long-Term Care for Thai Older Adults in Chiang Mai, Thailand: A Cluster Randomized Controlled Trial TCTR20190412004
}

Myo Nyein Aung ( $\square$ myo@juntendo.ac.jp)

Juntendo University School of Medicine Graduate School of Medicine: Juntendo Daigaku Igakubu Daigakuin Igaku Kenkyuka https://orcid.org/0000-0001-8175-6309

\section{Saiyud Moolphate}

Department of Public Health, Chiang Mai Rajabhat University

Thin Nyein Nyein Aung

Chiang Mai University Faculty of Medicine

\section{Yuka Koyanagi}

Tokyo Ariake University of Medical and Health Sciences

\section{Akraporn Kurusattra}

Department of Health Service Support, Ministry of Public Health

\section{Sutatip Chantaraksa}

Department of Health Service Support, Ministry of Public Health

\section{Siripen Supakankunti}

Chulalongkorn University Faculty of Economics

\section{Motoyuki Yuasa}

Department of Global Health Research, Juntendo University

\section{BMC Supplements Reviewed}

Keywords: global health, service delivery model, population ageing, health promotion, Asia, universal coverage, frailty, implementation research

Posted Date: January 27th, 2022

DOI: https://doi.org/10.21203/rs.3.rs-1275267/v1

License: (a) (i) This work is licensed under a Creative Commons Attribution 4.0 International License. Read Full License 


\section{Abstract}

Background Populations around the world are ageing faster, with the majority living in low- and middleincome countries where health and social care are yet to be universal and inclusive for the ageing population. This community-integrated intermediary care (CIIC) model is a novel prevention-based, long-term care model enhancing the family-based care system traditionally practiced in Thailand and neighboring Asian countries, and many low-and middle-income countries globally. This study assessed the effectiveness of the ClIC model in Chiang Mai, Thailand.

Method: The two-arm parallel intervention study was designed as a cluster randomized controlled trial. The study population at randomization and analysis was 2,788 participants: 1509 in 6 intervention clusters and 1279 in 6 control clusters. The research protocol was approved by the World Health Organization Ethics Review Committee WHO/ERC ID; ERC.0003064. The CIIC service intervention model is a combination of formal care and informal care in a subdistrict setting consisting of three components: (1) care prevention delivered as community group exercise and home exercise; (2) care capacity building of the family caregiver; and (3) community respite service. The primary outcome was family caregivers' burden at six months followup and secondary outcome was activity of daily living. Analysis applied the intention to treat approach using cluster level analysis via STATA 11 SE.

Result: Baseline characteristics did not differ between the two arms. Loss of follow up was $3.7 \%$. Mean age of the participants was 69.53 years. Females comprised 60\%. The COVID-19 pandemic caused delayed implementation. The proportion of families with reduced caregiver burden at six months follow-up was higher among the intervention clusters (mean $39.17 \%$ ) than control clusters (mean $28.62 \%$ ). The intervention clusters experienced less functional decline and fewer people with depression.

Discussion: When communities are integrated for preventing care, and families are empowered for giving care, it is possible to secure universal access to health and social care for the older persons, with basic resources mobilized from communities. This study had shown the CIIC model as an effective and potential step to the realization of universal health and long-term care coverage being inclusive of ageing populations in Thailand and globally.

Trial registration: It was registered at the Thailand Clinical Trial Registry-Trial registration number TCTR20190412004. https://www.thaiclinicaltrials.org/\#

\section{Introduction}

Population ageing, in which over 60 years of age is defined as old age, is increasing in Thailand. It was 0.7 million (7.2\%) in 2011, then rapidly increased to 11.5 million (almost $17 \%$ ) in 2020 , and is projected to be $33 \%$ by 2040.(1) This rapid demographic change has brought a steep rise in the burden of age-related morbidities and disabilities due to non-communicable diseases(2) Recent reports have shown that an increasing number of older Thais have become home-bound and bed-bound.(3) The scope of long-term care comprises medical and nursing care, personal care services, assistance services and social services, enabling people to live either independently or in residential settings when they can no longer carry out routine activities of daily living on 
their own.(4) Although health care services are universally accessible and publicly financed for all Thai citizens, Thailand still has some way to go to in terms of long-term care policy, due to the limited availability of formal care services and inequalities in access to those services. Such an imbalance poses the risk of pressure on hospital systems and excessive health care utilization as experienced in many countries.(4) Currently, long-term care in Thailand principally consists of informal care sectors which rely mainly on selfcare and families in terms of providing care and funding, as well as working in partnership with communities and volunteers.(5)

Families serve as the main caregivers for older Thai people. Filial responsibility is a social norm in Thai culture. $(6,7)$ There is intergenerational, two-way interdependency via financial remittance between adult children and grandparents raising grandchildren. (8) However, breadwinners and the middle-aged commonly migrate domestically for a better job and income, leaving their parents and the skipped generation families back at home in the rural area. As a result, nowadays there are few extended families still present in rural areas. With smaller family sizes and the increasing role of females in the labor market, the equilibrium of family care in Thailand is becoming a challenge to sustain. When families serve as the backbone of ageing care in the fast-ageing countries, the steeply increasing caregivers' burden negatively impacts on society through the loss of working hours, and even job loss, of caregivers. $(9,10)$ Policies supporting caregiving families are possible in terms of respite care, education and training, improving physical and mental wellbeing, family support programs, financial support, and family care leave.

A recent analysis from the same study site showed that the time consumed with caregiving negatively impacted on the career of one in four family caregivers. (11) Furthermore, caregiver burnout can lead to abuse of the older person.(12) Interventions promoting the care capacity within the informal care sector may relieve the burden on these families. Additionally, vastly differing levels of education and care competencies found amongst family caregivers may cause inequalities in the care of older people across lower-resource and lower-income countries. (13) There are regular courses for caregivers in Thailand, but family caregiving remains a grey area in terms of meeting the needs of recipients. An ideal technical guidance package would address the different needs and issues of the ageing persons. A recent study recruiting 867 older Thai couples and their family caregivers revealed that $5.5 \%$ of family caregivers were overburdened and needing respite. The median income of the study participants was 9000 Thai Baht. Respite service, in the form of community-integrated intermediary care, can be offered to family caregivers who become extremely burdened. $(14,15)$ A recent analysis showed that more than a quarter of family caregivers were willing to use respite services if they were accessible.(14)

Given the inevitable increasing burden on family caregivers, $(6,11,16)$ it is important to strengthen the traditional family-based long-term care model by interventions that empower families, along with care prevention programs to preserve the intrinsic capacity of the older persons. According to the World Health Organization, the concept of healthy ageing highlights the ability of older persons to do what they value doing, having intrinsic capacity and an age-friendly environment allowing for physical well-being, autonomy, social participation, and dignity. A recent survey in northern Thailand highlighted the profound need of care, with frailty (13.9\%) and pre-frailty (50.9\%) among the older persons.(17) The chance of frailty was higher amongst those with low-education and those living alone. This highlights the urgent need of care prevention 
in Thailand which has been overstretched for decades in terms of rehabilitation due to chronic diseases coupled with under-investment in active ageing.

Preventing frailty is possible though evidence-based community-integrated interventions.(18) Evidence in Japanese cohort studies showed that older people who participated in community-based group exercise programs were less likely to be frail, delaying the onset of dependency.(19) Community group exercise not only promotes physical well-being and prevention of care need but also represents the path to active ageing favoring autonomy, social connection and quality of the life.(20) Such outcomes are important for the Thai older population as they are either self-reliant or family dependent. Thailand communities are well-exposed to health promotion. The need is specifically to empower older community residents with a set of functional and suitable training exercises, comprising initial training of the techniques, time and space for community engagement and an affordable delivery model.

Universally accessible health and social care is an expectation of all second wave ageing countries like Thailand. In this study we created a novel ageing care model, that sought to enhance the family-based, longterm care system linked to primary health care services, integrated into the community and local government within each municipality. The community-integrated intermediary care (CIIC) model, serving as an intermediary center, was situated and integrated within the community. It was hoped that the CIIC model would link families and communities to local formal services and funding. In addition, it was intended to promote active ageing through a community-based care prevention exercise program for older persons. Moreover, the CIIC facility would serve as a small formal care home offering a short-term stay service by way of community-based respite care. The CIIC would deliver issue-specific, family caregivers' training and assistance to enhance their caregiving capacity. It would target integration at different levels: (1) micro level integration, among the older persons, their families, their peer group, and volunteers; (2) meso level integration, between the CIIC facility, the primary health care center, community stakeholders and the municipality office; and (3) macro level integration, between the public health and public administration sectors under different ministries in Thailand. (Table 1) Overall, the CIIC model sought to introduce an integrated package of formal long-term care services to the Thai community together with health promotion in order to boost family care capacity and prevent long term care needs. 
Table 1

Integration at different levels and resultant action or outcomes in the community-integrated intermediary care (CIIC) model

\begin{tabular}{|c|c|c|}
\hline $\begin{array}{l}\text { Level of } \\
\text { integration }\end{array}$ & Integration & Action or outcome \\
\hline Micro & $\begin{array}{l}\text { Older persons } \\
\text { Family caregivers } \\
\text { Volunteers exercise trainer } \\
\text { Health Volunteers }\end{array}$ & $\begin{array}{l}\text { 1. Home exercise } \\
\text { 2. Community-based, group care prevention } \\
\text { functional exercise }\end{array}$ \\
\hline Meso & $\begin{array}{l}\text { CIIC facility } \\
\text { Primary care center staff } \\
\text { Community stake holders } \\
\text { Municipality office }\end{array}$ & $\begin{array}{l}\text { 1. Care capacity building } \\
\text { 2. Respite center and referrals } \\
\text { 3. Referrals } \\
\text { 4. Home visits for training family caregivers } \\
\text { 5. Coordinating community-based activities } \\
\text { 6. Data collection }\end{array}$ \\
\hline Macro & $\begin{array}{l}\text { Public health authority } \\
\text { Public Administration authority } \\
\text { University academics } \\
\text { World Health Organization (WHO) } \\
\text { Japan International Cooperation } \\
\text { Agency (JICA) }\end{array}$ & $\begin{array}{l}\text { 1. Building CIIC facility } \\
\text { 2. Establishment, implementation, and } \\
\text { sustainability of the model } \\
\text { 3. Funding } \\
\text { 4. Research }\end{array}$ \\
\hline
\end{tabular}

Whether the presence of a community-integrated intermediary care center in a district can reduce the burden of family caregivers and promote the functional ability and quality of life (QOL) of older adults in the communities of Thailand is an interesting research question that has not yet been researched systematically. This study aimed to assess the efficacy of a community-integrated intermediary care (CIIC) model and compare it to the existing traditional family care model in Thailand. The objective of this study, therefore, was to assess the effectiveness of a community-integrated intermediary care (CIIC) service model that assisted families' provision of long-term care for older adults in terms of the primary outcome, the family caregivers' burden, and secondary outcomes, the older persons' functional ability determined by their activities of daily living and quality of life.

\section{Materials And Methods}

\subsection{Design, setting and participants}


The two-arm parallel intervention study was designed as a cluster randomized controlled trial. It recruited 2,788 participants: 1509 participants in 6 intervention clusters and 1279 participants in 6 control clusters. The research protocol was approved by the World Health Organization Ethics Review Committee WHO/ERC ID; ERC.0003064, dated 7 March 2019 and Boromrajonani College of Nursing, Lampang Thailand Ethics Review Committee E2562/005, dated 4 March 2019. It was registered at the Thailand Clinical Trial Registry, Trial registration number TCTR20190412004.(21) The trial was conducted over a period of 2 years. The net intervention period was six months.

\section{Study setting}

The trial location was in Chiang Mai, Northern Thailand. A total of 284,457 older adults resided in Chiang Mai city according to a provincial report in 2019 . This number accounts for $18 \%$ of its population and was higher than the national proportion of people aged 60 years and older, which was $16 \%$. The culture in Chiang Mai is similar to that of many neighbouring ASEAN countries in terms of caring for older parents as a family tradition and social value.

Two subdistricts were involved in the study: subdistrict XXX (10 villages) for the intervention arm, and subdistrict YYY (15 villages) for the control arm. They are in the same Mueang Chiang Mai district of Chiang Mai province. The distance between the two subdistricts were far enough to prevent relayed messages although they were of a similar demographic and socio-cultural characteristics. The two subdistricts have a similar population (more than 18,000), and similar ageing rates (more than $18 \%$ of people over 60 ). Residents in both subdistricts are mostly Thai people who have access to health care services under the national health insurance system and social welfare services for older people covered by municipality funds.

\section{Sample size and power}

The estimated sample was 1500 participants in each arm to determine the size difference of 0.5 unit with standard deviation (SD) of 4 between the two arms. STATA version 11SE (Stata Corporation, College Station, TX) was utilized for sample and power estimations. The precision levels applied are a P-value of 0.05 with a $95 \%$ confidence interval. The sample size was inflated for design effect 1.2 of cluster randomized design application, and for compensation of potential non-responses and drop-outs during the recruitment and study of up to $20 \%$. Moreover, the sample size was estimated to be sufficient for detecting minimal difference in key parameters such as mean $\mathrm{CBI}, \mathrm{ADL}$, and health-related $\mathrm{QOL}$ indicators within the study population. We calculated backward sample-power calculation at the end.

\section{Sequence generation, random allocation, and blinding}

Cluster randomization was used to prevent contamination between the intervention subdistrict and control subdistrict. It was not possible to randomize the villages from different administrative areas, municipalities, and directly allocate them to the intervention and control arms. Therefore, we practiced internal randomization to recruit six eligible clusters randomly within the intervention arm municipality, which has 1015 villages, and likewise, in the control arm. (22) (Figure 1) 
A statistician blinded to the study generated a random number for each arm of the study and recruited villages randomly within each arm. The control arm and intervention arm villages were geographically distant and administratively exclusive, yet they were in the same province and similar demographically, socially and culturally in order for social intervention evaluation.

It was not possible to do double blinding. However, participants were blind to allocation. Participants and research assistants carrying out assessment were not able to know the randomly-allocated clusters before the study begun. Thus, bias and contamination were controlled.

The unit of randomization, cluster, was a village. Participants in the intervention clusters received the community-integrated intermediary care (CIIC) facility and service intervention, after screening for eligibility. Eligibility criteria for a cluster was a village which had more than 300 older persons over 60 years of age, at the time of randomization.

\section{Inclusion criteria for study participants}

1. Persons over 60 years of age and their family caregivers

2. Either male or female

3. Resident in the study site districts

\subsection{Exclusion criteria}

1. Persons over 60 or their family caregivers without informed consent

2. Persons who could not understand the explanation for informed consent although provided with language support

3. Households without an older person over 60 years

4. People with cognitive impairment or severe impairment decision-making abilities

\subsection{Outcome measurement}

Validated instruments commonly used in ageing and long-term care research were carefully chosen in order to assess the impact of the intervention in objective outcomes. Most of the instruments used were already translated into Thai versions and validated in previous studies and programs in Thailand. We conducted a pilot test for the target population and ensured reliability of all the instruments in the study setting and context.

\section{Primary outcome}

Primary outcome was the family caregiver's burden at the six months follow-up. The caregiver burden inventory (CBI) was used to measure burden of the caregiver. $(23,24)$ The $\mathrm{CBI}$ was applied to measure the 
family caregiver's burden at baseline month 0 and month 6 . The CBI is an internationally validated, 24-item, 5point Likert scale which measures the burden of caregiver in 5 dimensions as follows:

a. Time dependence burden (5 items)

b. Developmental burden (5 items)

c. Physical burden (4 items)

d. Social burden (5 items)

e. Emotional burden (4 items).

After summing up the total score, a score greater than 36 indicates a risk of burnout and a score near 24 indicates a need to seek respite care.

\section{Secondary outcomes}

Secondary outcomes consisted of biopsychosocial indictors such as functional ability, depression and quality of life of the elderly, measured comparing the intervention and control arms after the first six months of intervention. Functional ability was assessed by applying Barthel's Activity of Daily Living (ADL) assessment. (25) ADL measures the level of ability for 10 basic items (bowels, bladder, grooming, toileting, feeding, transfer, mobility, dressing, stairs, bathing). Total possible scores range from $0-20$, with lower scores indicating increased disability. Depression was screened by applying the Geriatric Depression Scale (GDS), which is commonly-used internationally, validated and regularly used in Thailand. (26) Health-related quality of life was measured through EQ 5D questionnaires. (27) A validated Thai version instrument was readily available and used after piloting. Health-related quality of life (EQ-5D-5L) with EQ visual analogue scale (EQ VAS) $(27,28)$ was applied to measure the health-related quality of life of the elderly persons. The EQ-5D-5L comprises five dimensions: mobility, self-care, usual activities, pain/discomfort and anxiety/ depression. Each dimension has 5 levels. The EQ VAS is a measure of overall self-rated health status. It records the respondent's self-rated health on a vertical, visual analogue scale where the endpoints are labelled 'Best imaginable health state' and 'Worst imaginable health state'.

\subsection{Intervention}

The community-integrated intermediary care (CIIC) model is a novel prevention-based, long-term care model created to enhance the family-based long-term care system (Figure 2) which has traditionally been practiced in Thailand and its neighboring Asian countries as well as many low-and middle-income countries globally. $(14,29)$ Conceptually it is a combination of formal care and informal care in a district, or subdistrict or a city. To implement the CIIC service model a community-based facility had to be established within the community, mobilizing community resources and the municipality funds. It would serve as a five to ten beds respite home and office for CIIC staffs. Two auxiliary nurses would work alternatively for care, coordination, and capacity-building with two assistants and volunteers. The CIIC facility would be geographically communitycentered, functionally linked to the primary health care center and administratively linked to the municipality 
office. However, its human resources and professionals would be separate, not from the primary health care center. The CIIC service model consisted of three components: (1) care prevention exercise, (2) care capacity building of the family caregiver; and (3) community respite service.

Care prevention exercise is to prevent the long-term care need of the older persons, to preserve their functional ability leading to active ageing. (Figure 2) It is a set of functional training exercises particularly designed for older adults either in a sitting or standing position. We provided two options: (a) 45 minutes long communitybased group exercise; and (b) 10 minutes long home exercise set for every day of the week to be practiced twice a day individually or with friends and family at home. We provided an exercise DVD, wall posters to guide the exercise techniques, and a calendar for recording exercise. Home sets of exercises consisted of stretch, upper limb, lower limb, trunk and squat components (30), whereas group exercise consisted of dynamic and static stretch, tube training for upper and lower limbs, squat, balance training, walking and brain training. Community-based group exercise activity required a venue, schedule, and a television or projector for a group of 30 persons. We created six such venues in the intervention clusters. Before launching the community-based group exercise, community volunteers were trained to be the leaders of group exercise. The training took four days. Stakeholders made the schedule of group exercises depending on the participant's available time.

Secondly, CIIC provided training and assistance to enhance the family caregiver's care capacity. (Figure 2) The training was not a general, regular course. It was an issue-based technical training, relating to the need of the care recipient. CIIC teams arranged home visits when caring families made a request. A care expert nurse and the team reviewed the care techniques and taught the family caregiver on site. This activity was linked to the primary health care service, and community health volunteers coordinated with the municipality authority. A visit to home screen the care need of the older persons, the burden of the family caregiver and technical gaps, and the home modification need indicated what could be provided by the municipality and what could be referred to the primary health care center.

The third service of CIIC was to provide formal long-term care service in the form of a community respite home for eligible older persons. (Figure 2) Eligibility criteria were set by the city stakeholders. In this study the eligibility criteria for the CIIC temporary respite care service was for applicants with full-time, unpaid family caregivers caring for dependent individuals over 60 years of age and meeting the eligibility as assessed using the $A D L$ and $C B I .(14)$

\section{ADL Criteria}

In order to be eligible to be admitted to the CIIC facility, the family caregiver had to be personally providing the care recipient with assistance for at least 2 of the following 6 activities of daily living (ADL): 1) Bathing-the family caregiver is assisting the older adult with bathing, including help with washing, shampooing, getting in or out of the tub or shower, brushing teeth, and other aspects of personal grooming (Bathing ADL score 0 ); 2) Dressing- the family caregiver is assisting the older adult with dressing, including helping the individual put on or take off clothing and footwear (Dressing ADL score 0 or 1); 3) Toileting-the family caregiver is helping the older adult get on or off the toilet, commode, or bedpan as well as clean themselves, or the individual is incontinent (Toileting ADL score 0 or 1 ); 4) Transferring- the family caregiver is helping the older adult get to 
and from a bed or chair (Transfer ADL score 0 or 1 ); 5) Walking or mobility- the family caregiver is helping the older adult move from one stationary point to another by removing obstacles, opening doors, and assisting with canes, wheelchairs, or other assistive devices (Mobility ADL score 0 or 1 ); 6) Eating or feeding- the family caregiver is helping the older adult who has difficulty chewing or swallowing without assistance or needs partial or total help with eating (Feeding ADL 0 or 1 ).

\section{CBI Criteria}

On the assessment of caregiver burden, the following 2 eligibility criteria were required to utilize the respite care service: 1 ) time dependency items score $>17$; and 2 ) physical health items score $>14$. If there was only one person in the family acting as a caregiver for the older adult with the qualifying ADL criteria and the caregiver had to leave the house to travel, the older adult would qualify for admission to the CIIC based on the available capacity of the facility on the appointment days. These criteria could be revised by stakeholders depending on the estimates of the population to be served, budget of the municipality, and capacity of the facility.

\section{Control group}

The CIIC intervention was evaluated in comparison with an active comparator which was the existing service in Thailand. Control arm participants received the usual care (ie, the current system of LTC common to all provinces in Thailand), consisting principally of a health volunteers' assistance to those living alone or severely dependent older persons in addition to traditional family-based care. The difference between the services of the 2 arms refers to the newly-launched CIIC services, as described above.

\section{Screening and Assessment of Family Burden and LTC Capacity}

All older adults and caregivers in each cluster who provided informed consent were screened for ADL status and health-related QOL utilizing the EuroQol 5-dimensions 5-levels (EQ-5D-5L) for older adults, and caregiver burden measured using the Caregiver Burden Inventory (CBI). In both arms, a basic health check, blood pressure check, and BMI assessment were provided by the study participants. If any disease was suspected through the health check, appropriate referrals to existing health care services were provided. This was a benefit for all study participants. The control group also received the same assessment with an explanation that the assessment was being conducted as part of a survey and the result would be utilized for research purposes. They also received the benefit of the additional health check followed by appropriate referrals to health care professionals.

\subsection{Data analysis}

All analysis applied cluster level analysis for all outcome measures. Cluster adjusted t-test and cluster adjusted chi-squared test were applied to compare the outcome between the intervention and control groups. At first the result of the baseline survey was compared between the intervention and control arm clusters to check the balance between the two arms and to detect possible confounders. (Table 2)We compared the level 
of the indicators measured at the baseline, applying cluster level analysis. Similarity between the two arms after randomization indicated that cluster adjusted analysis was sufficient.

Intention-to treat analysis was applied. Bio, psycho, social indicators were compared between the two arms. The primary outcome was the caregiver burden inventory (CBI). (Table 3-5)

First, we compared outcomes in the categorical data. The CBI scores were categorised into three groups: a low burden (<24), a medium burden (24-36) and a high burden $(>36)$.

Second, we compared the change in CBI level between the baseline level and evaluation level. The decline in CBI within six months follow-up was defined as a desirable outcome event.(Table 4) In this analysis, those lost to follow-up or died were treated as a negative outcome in the intention to treat approach. Finally, we compared the level of the indicator, $\mathrm{CBI}$ measured at the evaluation, applying cluster level analysis. The power of the sample was 0.79 for the primary outcome results.

Effectiveness in preventing care need or functional decline was analysed using the secondary outcome ADL. (Table 6-8) First it was analysed in three categories. Then the level of ADL was compared between intervention and control clusters. The change in ADL level was used to detect the outcome functional decline. Sustaining the same level of $A D L$ or an increase in ADL level within six months of follow up was defined as desirable outcome. (Table 7) This analysis also applied intention to treat approach and cluster level analysis. Another secondary outcome GDS was analysed in categories and also comparing the level of GDS. (Table 9 and 10) Health related QOL was analysed comparing EQ5D5L level between the intervention and control arms in cluster level analysis. (Table 11)

\section{Results}

The number participants at randomization were 1509 in the intervention arm and 1279 in the control arm. The number of participants at the analysis were 1460 (95.92\%) in the intervention arm and $1250(96.65 \%)$ in the control arm. A total of 49 (3.25\%) people died in the intervention arm and 29 in the control arm (2.27\%). The overall loss of follow-up was $100(3.7 \%)$ people because of movement to other regions and loss of contact.

The mean age of the study participants was 69.53 years with females representing sixty percent of the participants. $10.3 \%$ of the participants were living alone, while $33.9 \%$ were staying together with their spouse, $42.9 \%$ with children and $5.3 \%$ with siblings. Sixty-two percent of the participants were primary school educated, $31 \%$ achieved secondary and tertiary level education, whilst only $7 \%$ had no formal education. Thirty percent of the participants were still working in an employed jobBaseline characteristics

When comparing baseline levels of $\mathrm{CBI}, \mathrm{ADL}$ and GDS between the intervention and control arms, there were no significant differences ( $p=0.08$ MANOVA). Randomization was successful. We analysed all three outcomes in the MANOVA model, and they were not statistically significant.

Table 2. Baseline characteristic level of outcome indicator after randomization, by clusters in intervention and control arms 


\begin{tabular}{|c|c|c|c|c|c|c|c|c|c|}
\hline & Cluster & CBI & & $A D L$ & & GDS & & $E Q$ ind & \\
\hline & & Mean & SD & Mean & SD & Mean & SD & Mean & SD \\
\hline \multirow[t]{7}{*}{ Intervention } & 1 & 2.81 & 6.38 & 19.28 & 2.48 & 1.35 & 2.25 & 0.83 & 0.20 \\
\hline & 2 & 14.36 & 13.16 & 19.43 & 1.97 & 2.11 & 2.32 & 0.72 & 0.23 \\
\hline & 3 & 2.36 & 6.09 & 19.34 & 2.30 & 1.03 & 2.13 & 0.86 & 0.20 \\
\hline & 4 & 1.48 & 4.67 & 19.23 & 2.30 & 1.14 & 1.99 & 0.89 & 0.16 \\
\hline & 5 & 1.56 & 7.21 & 19.40 & 2.45 & 0.78 & 1.30 & 0.85 & 0.23 \\
\hline & 6 & 6.63 & 9.83 & 18.76 & 3.17 & 2.76 & 2.69 & 0.75 & 0.28 \\
\hline & Mean & 4.37 & 9.07 & 19.23 & 2.50 & 1.57 & 2.29 & 0.81 & 0.23 \\
\hline \multirow[t]{7}{*}{ Control } & 7 & 4.43 & 8.61 & 18.99 & 3.21 & 1.44 & 2.31 & 0.78 & 0.26 \\
\hline & 8 & 4.36 & 9.17 & 19.40 & 2.15 & 0.92 & 1.58 & 0.81 & 0.21 \\
\hline & 9 & 4.69 & 9.94 & 19.33 & 2.34 & 0.85 & 1.46 & 0.84 & 0.16 \\
\hline & 10 & 3.80 & 10.00 & 19.52 & 1.85 & 1.06 & 1.60 & 0.83 & 0.25 \\
\hline & 11 & 3.90 & 9.31 & 19.06 & 2.76 & 0.97 & 1.61 & 0.78 & 0.24 \\
\hline & 12 & 3.66 & 8.61 & 19.21 & 2.24 & 2.13 & 3.09 & 0.79 & 0.24 \\
\hline & Mean & 4.15 & 9.46 & 19.25 & 2.46 & 1.23 & 2.07 & 0.81 & 0.23 \\
\hline icc & & 0.052 & & 0.017 & & 0.019 & & 0.047 & \\
\hline$P$ & & 0.67 & & 0.69 & & 0.35 & & 0.79 & \\
\hline MANOVA P value & 0.08 & & & & & & & & \\
\hline
\end{tabular}

CBI: Caregiver Burden Inventory scale, ADL: Activity of Daily Living score, GDS: Geriatric Depression Scale, EQ5D5L: European Quality of Life scale, icc: Inter-cluster correlation, P: p-value of MANOVA test, SD: standard deviation

\section{Primary Outcome: effectiveness in reducing the caregiver burden $\mathrm{CBI}$}

Effectiveness of the intervention was assessed applying the $\mathrm{CBI}$ as the primary outcome of the study.

1. The proportion of high-burdened families ( $\mathrm{CBI}$ score $\geq 36$ ) was higher in the control arm clusters than the intervention arm clusters. It was statistically significant $(P<0.001$, icc 0.03 , cluster-adjusted Chi-squared test). (Table 3)

Table 3 Caregiver burden by clusters in intervention and control arms 


\begin{tabular}{|c|c|c|c|c|c|c|c|c|c|c|c|c|c|}
\hline & Clusters & Interv & tion arr & & & & & Cont & arm & & & & \\
\hline & & Less & urden & $\begin{array}{l}\text { Mor } \\
\text { Burc }\end{array}$ & & & & Less & urden & $\begin{array}{l}\text { Mo } \\
\text { Bur }\end{array}$ & & & \\
\hline & & UDK & & $24-$ & & $\geq 3$ & & DDK & & 24 & & $\geq 3$ & \\
\hline & & $\mathrm{n}$ & $\%$ & $\mathrm{n}$ & $\%$ & $\mathrm{n}$ & $\%$ & $\mathrm{n}$ & $\%$ & $\mathrm{n}$ & $\%$ & $\mathrm{n}$ & $\%$ \\
\hline & 1 & 119 & 98.35 & 1 & 0.83 & 1 & 0.83 & & & & & & \\
\hline & 2 & 90 & 76.92 & 22 & 18.80 & 5 & 4.27 & & & & & & \\
\hline & 3 & 208 & 98.11 & 3 & 1.42 & 1 & 0.47 & & & & & & \\
\hline & 4 & 144 & 98.63 & 2 & 1.37 & 0 & 0.00 & & & & & & \\
\hline & 5 & 144 & 97.96 & 1 & 0.68 & 2 & 1.36 & & & & & & \\
\hline & 6 & 110 & 88.71 & 13 & 10.48 & 1 & 0.81 & & & & & & \\
\hline & 7 & & & & & & & 44 & 93.62 & 3 & 6.38 & 0 & 0.00 \\
\hline & 8 & & & & & & & 143 & 96.62 & 2 & 1.35 & 3 & 2.03 \\
\hline & 9 & & & & & & & 188 & 97.92 & 1 & 0.52 & 3 & 1.56 \\
\hline & 10 & & & & & & & 178 & 95.70 & 2 & 1.08 & 6 & 3.23 \\
\hline & 11 & & & & & & & 121 & 96.03 & 2 & 1.59 & 3 & 2.38 \\
\hline & 12 & & & & & & & 103 & 96.26 & 1 & 0.93 & 3 & 2.80 \\
\hline icc & $0.0399 \mathrm{Cl}$ & 0.001 & $0.0788)$ & & & & & & & & & & \\
\hline$P$ & 0.0001 & & & & & & & & & & & & \\
\hline
\end{tabular}

CBI: Caregiver Burden Inventory scale, icc: Inter-cluster correlation, Cl: 95\% confidence interval, P: p- value of cluster adjusted Chi-squared test

2. The change in the $\mathrm{CBI}$ was assessed according to the proportion of families whose $\mathrm{CBI}$ was reduced by the intervention. (Table 4) The proportion of families with a reduced caregiver burden at the six months follow-up was higher among the intervention clusters (mean $39.17 \%$ ) than the control clusters (mean $28.62 \%$ ). It was statistically significant ( $P<0.001$, icc 0.01 , cluster adjusted Chi-squared test). 
Table 4 Proportion of families with reduced CBI, by clusters in intervention and control arms

\begin{tabular}{|c|c|c|c|c|c|c|c|c|c|}
\hline & Clusters & Interv & ttion arn & & & Cont & & & \\
\hline & & $\mathrm{CBI}$ & & $\mathrm{CBI}$ & & $\mathrm{CBI}$ & & CBI & \\
\hline & & Not $r$ & uced & Redı & & Not $r$ & & Redu & \\
\hline & & $\mathrm{n}$ & $\%$ & $\mathrm{n}$ & $\%$ & $\mathrm{n}$ & $\%$ & $\mathrm{n}$ & $\%$ \\
\hline & 1 & 175 & 66.04 & 90 & 33.96 & & & & \\
\hline & 2 & 157 & 64.61 & 86 & 35.39 & & & & \\
\hline & 3 & 96 & 38.10 & 156 & 61.90 & & & & \\
\hline & 4 & 166 & 72.17 & 64 & 27.83 & & & & \\
\hline & 5 & 117 & 50.00 & 117 & 50.00 & & & & \\
\hline & 6 & 207 & 72.63 & 78 & 27.37 & & & & \\
\hline & 7 & & & & & 198 & 90.83 & 20 & 9.17 \\
\hline & 8 & & & & & 114 & 55.88 & 90 & 44.12 \\
\hline & 9 & & & & & 110 & 51.89 & 102 & 48.11 \\
\hline & 10 & & & & & 145 & 63.32 & 84 & 36.68 \\
\hline & 11 & & & & & 175 & 84.13 & 33 & 15.87 \\
\hline & 12 & & & & & 171 & 82.21 & 37 & 17.79 \\
\hline icc & $0.0994 \mathrm{Cl}$ & $0.02,0$ & & & & & & & \\
\hline$P$ & $<0.001$ & & & & & & & & \\
\hline
\end{tabular}

CBI: Caregiver Burden Inventory scale, icc: Inter-cluster correlation, Cl: 95\% confidence interval, P: p- value of cluster adjusted Chi-squared test

3. We compared the level of $\mathrm{CBI}$ between the intervention and control arms at the evaluation, at six months and after the intervention. The difference in the levels of $\mathrm{CBI}$ between the intervention and the control clusters was statistically not significant ( $P=0.68$, icc 0.05 cluster-adjusted t-test). (Table 5 ) It was also not significant statistically in the GEE model. 
Table $5 \mathrm{CBI}$ levels by clusters in intervention and control arms

\begin{tabular}{|c|c|c|c|c|c|}
\hline & \multirow[t]{2}{*}{ Clusters } & \multicolumn{2}{|c|}{ Intervention arm } & \multicolumn{2}{|c|}{ Control arm } \\
\hline & & Mean & SD & Mean & SD \\
\hline & 1 & 2.13 & 5.86 & & \\
\hline & 2 & 7.13 & 12.76 & & \\
\hline & 3 & 2.23 & 4.75 & & \\
\hline & 4 & 4.72 & 8.99 & & \\
\hline & 5 & 0.85 & 3.86 & & \\
\hline & 6 & 4.97 & 9.84 & & \\
\hline & Mean & 3.39 & 8.04 & & \\
\hline & 7 & & & 3.07 & 7.48 \\
\hline & 8 & & & 1.07 & 2.57 \\
\hline & 9 & & 1.54 & 3.90 & \\
\hline & 10 & & & 4.03 & 9.54 \\
\hline & 11 & & & 4.66 & 10.45 \\
\hline & 12 & & & 3.72 & 7.31 \\
\hline & Mean & & & 2.92 & 7.38 \\
\hline icc & 0.0527 & & & & \\
\hline$P$ & 0.68 & & & & \\
\hline
\end{tabular}

CBI: Caregiver Burden Inventory scale, icc: Inter-cluster correlation, P: p-value of cluster adjusted Chi-squared test, SD: standard deviation

\section{Secondary outcomes}

Effectiveness in preventing functional decline.

1. The proportion of $A D L$ decline was higher in the control arm clusters than in the intervention arm clusters. It was statistically significant ( $P=0.004$, icc 0.001 , cluster-adjusted Chi-squared test). (Table 6$)$

Table 6 ADL among three groups in the intervention and control clusters 


\begin{tabular}{|c|c|c|c|c|c|c|c|c|c|c|c|c|c|}
\hline & \multirow[t]{3}{*}{ Clusters } & \multicolumn{6}{|c|}{ Intervention arm } & \multicolumn{6}{|c|}{ Control Arm } \\
\hline & & \multicolumn{2}{|c|}{$\mathrm{ADL} \geq 12$} & \multicolumn{2}{|c|}{$5-11$} & \multicolumn{2}{|c|}{$0-4$} & \multicolumn{2}{|c|}{$\mathrm{ADL} \geq 12$} & \multicolumn{2}{|c|}{$5-11$} & \multicolumn{2}{|c|}{$0-4$} \\
\hline & & $\mathrm{n}$ & $\%$ & $\mathrm{n}$ & $\%$ & $\mathrm{n}$ & $\%$ & $\mathrm{n}$ & $\%$ & $\mathrm{n}$ & $\%$ & $\mathrm{n}$ & $\%$ \\
\hline & 1 & 229 & 97.03 & 3 & 1.27 & 4 & 1.69 & & & & & & \\
\hline & 2 & 220 & 96.07 & 4 & 1.75 & 5 & 2.18 & & & & & & \\
\hline & 3 & 237 & 95.95 & 9 & 3.64 & 1 & 0.40 & & & & & & \\
\hline & 4 & 215 & 99.08 & 0 & 0.00 & 2 & 0.92 & & & & & & \\
\hline & 5 & 218 & 97.32 & 4 & 1.79 & 2 & 0.89 & & & & & & \\
\hline & 6 & 242 & 93.80 & 8 & 3.10 & 8 & 3.10 & & & & & & \\
\hline & 7 & & & & & & & 202 & 95.28 & 4 & 1.89 & 6 & 2.83 \\
\hline & 8 & & & & & & & 169 & 96.02 & 5 & 2.84 & 2 & 1.14 \\
\hline & 9 & & & & & & & 191 & 95.98 & 7 & 3.52 & 1 & 0.50 \\
\hline & 10 & & & & & & & 213 & 96.82 & 6 & 2.73 & 1 & 0.45 \\
\hline & 11 & & & & & & & 183 & 93.85 & 11 & 5.64 & 1 & 0.51 \\
\hline & 12 & & & & & & & 182 & 92.39 & 15 & 7.61 & 0 & 0.00 \\
\hline icc & $0.0014 \mathrm{Cl}$ & $(0.000$ & 64) & & & & & & & & & & \\
\hline $\mathrm{P}$ & 0.0035 & & & & & & & & & & & & \\
\hline
\end{tabular}

ADL: Activity of Daily Living score, icc: Inter-cluster correlation, Cl: $95 \%$ confidence interval, P: $p$ - value of cluster adjusted Chi-squared test

2. Change in ADL: The proportion of participants without functional decline at the six months follow-up was higher among the intervention clusters (mean 83\%) than the control cluster (mean 78\%). The preventive effect was statistically significant ( $P=0.004$, icc 0.001 , cluster-adjusted Chi-squared test). (Table 7$)$

Table 7 Proportion without ADL decline among the intervention and control clusters 


\begin{tabular}{|c|c|c|c|c|c|c|c|c|c|}
\hline & \multirow[t]{3}{*}{ Clusters } & \multicolumn{4}{|c|}{ Intervention arm } & \multicolumn{4}{|c|}{ Control arm } \\
\hline & & \multicolumn{2}{|c|}{ ADL declined } & \multicolumn{2}{|c|}{ Care prevented } & \multicolumn{2}{|c|}{ ADL declined } & \multicolumn{2}{|c|}{ Care prevented } \\
\hline & & $\mathrm{n}$ & $\%$ & $\mathrm{n}$ & $\%$ & $\mathrm{n}$ & $\%$ & $\mathrm{n}$ & $\%$ \\
\hline & 1 & 46 & 17.36 & 219 & 82.64 & & & & \\
\hline & 2 & 42 & 17.28 & 201 & 82.72 & & & & \\
\hline & 3 & 49 & 19.44 & 203 & 80.56 & & & & \\
\hline & 4 & 23 & 10.00 & 207 & 90.00 & & & & \\
\hline & 5 & 33 & 14.10 & 201 & 85.90 & & & & \\
\hline & 6 & 67 & 23.51 & 218 & 76.49 & & & & \\
\hline & 7 & & & & & 53 & 24.31 & 165 & 75.69 \\
\hline & 8 & & & & & 40 & 19.61 & 164 & 80.39 \\
\hline & 9 & & & & & 30 & 14.15 & 182 & 85.85 \\
\hline & 10 & & & & & 47 & 20.52 & 182 & 79.48 \\
\hline & 11 & & & & & 53 & 25.48 & 155 & 74.52 \\
\hline & 12 & & & & & 52 & 25.00 & 156 & 75.00 \\
\hline icc & \multicolumn{9}{|c|}{$0.0107, \mathrm{Cl}(0.0000,0.0232)$} \\
\hline $\mathrm{P}$ & \multicolumn{9}{|l|}{0.0043} \\
\hline
\end{tabular}

ADL: Activity of Daily Living score, icc: Inter-cluster correlation, Cl: $95 \%$ confidence interval, P: p- value of cluster adjusted Chi-squared test

3. The difference in the levels of $A D L$ between the intervention and the control clusters was statistically not significant ( $P=0.069$, icc 0.017 , cluster-adjusted t-test). (Table 8 ) It was also not significant statistically in the GEE model.

Table 8 ADL levels by clusters in intervention and control arms 


\begin{tabular}{|c|c|c|c|c|c|}
\hline & \multirow[t]{2}{*}{ Clusters } & \multicolumn{2}{|c|}{ Intervention arm } & \multicolumn{2}{|c|}{ Control arm } \\
\hline & & Mean & SD & Mean & SD \\
\hline & 1 & 17.06 & 6.49 & & \\
\hline & 2 & 18.00 & 5.31 & & \\
\hline & 3 & 18.58 & 3.74 & & \\
\hline & 4 & 18.41 & 4.96 & & \\
\hline & 5 & 18.56 & 4.50 & & \\
\hline & 6 & 16.78 & 6.44 & & \\
\hline & Mean & 17.85 & 5.43 & & \\
\hline & 7 & & & 18.19 & 4.33 \\
\hline & 8 & & & 16.27 & 6.97 \\
\hline & 9 & & & 18.07 & 5.08 \\
\hline & 10 & & & 18.33 & 4.31 \\
\hline & 11 & & & 17.52 & 5.20 \\
\hline & 12 & & & 17.48 & 4.97 \\
\hline & Mean & & & 17.67 & 5.23 \\
\hline icc & 0.0173 & & & & \\
\hline$P$ & 0.69 & & & & \\
\hline
\end{tabular}

ADL: Activity of Daily Living score, icc: Inter-cluster correlation, P: p value of cluster adjusted Chi-squared test, SD: standard deviation

\section{Effectiveness in preventing depression.}

1. The proportion of participants without depression was higher among the intervention clusters (mean $87.14 \%)$ than the control clusters (85.89\%). It was statistically significant $(P<0.001$, icc 0.02 , cluster-adjusted Chi-squared test) (Table 9).

Table 9 State of depression GDS three groups in two clusters 


\begin{tabular}{|c|c|c|c|c|c|c|c|c|c|c|c|c|}
\hline \multirow[t]{3}{*}{ Clusters } & \multicolumn{6}{|c|}{ Intervention arm } & \multicolumn{6}{|c|}{ Control Arm } \\
\hline & \multicolumn{2}{|c|}{ Normal 0-4 } & \multicolumn{2}{|c|}{$\begin{array}{l}\text { Mild } \\
\text { depression } \\
5-10\end{array}$} & \multicolumn{2}{|c|}{$\begin{array}{l}\text { Severe } \\
\text { depression } \\
11-15\end{array}$} & \multicolumn{2}{|c|}{ Normal 0-4 } & \multicolumn{2}{|c|}{$\begin{array}{l}\text { Mild } \\
\text { depression } \\
5-10\end{array}$} & \multicolumn{2}{|c|}{$\begin{array}{l}\text { Severe } \\
\text { depression } \\
11-15\end{array}$} \\
\hline & $\mathrm{n}$ & $\%$ & $n$ & $\%$ & $\mathrm{n}$ & $\%$ & $\mathrm{n}$ & $\%$ & $\mathrm{n}$ & $\%$ & $\mathrm{n}$ & $\%$ \\
\hline 1 & 221 & 93.64 & 15 & 6.36 & 0 & 0.00 & & & & & & \\
\hline 2 & 196 & 86.34 & 31 & 13.66 & 0 & 0.00 & & & & & & \\
\hline 3 & 224 & 90.69 & 23 & 9.31 & 0 & 0.00 & & & & & & \\
\hline 4 & 186 & 86.11 & 29 & 13.43 & 1 & 0.46 & & & & & & \\
\hline 5 & 203 & 90.63 & 21 & 9.38 & 0 & 0.00 & & & & & & \\
\hline 6 & 197 & 76.36 & 59 & 22.87 & 2 & 0.78 & & & & & & \\
\hline 7 & & & & & & & 180 & 84.91 & 24 & 11.32 & 8 & 3.77 \\
\hline 8 & & & & & & & 159 & 90.34 & 15 & 8.52 & 2 & 1.14 \\
\hline 9 & & & & & & & 171 & 86.36 & 16 & 8.08 & 11 & 5.56 \\
\hline 10 & & & & & & & 195 & 88.64 & 17 & 7.73 & 8 & 3.64 \\
\hline 11 & & & & & & & 171 & 87.69 & 15 & 7.69 & 9 & 4.62 \\
\hline
\end{tabular}


icc $\quad 0.0218(0.0002,0.0434)$

$\mathrm{P} \quad<0.0001$

GDS: Geriatric Depression Scale, icc: Inter-cluster correlation, Cl: 95\% confidence interval, P: p-value of cluster adjusted Chi-squared test

2. The level of GDS was not significantly different between the intervention and control clusters $(P=0.23$, icc 0.03 , cluster-adjusted t-test). (Table 10)

Table 10 The level of GDS by clusters in intervention and control arms

\begin{tabular}{|c|c|c|c|c|c|}
\hline & \multirow[t]{2}{*}{ Clusters } & \multicolumn{2}{|c|}{ Intervention arm } & \multicolumn{2}{|c|}{ Control arm } \\
\hline & & Mean & SD & Mean & SD \\
\hline & 1 & 3.28 & 4.41 & & \\
\hline & 2 & 3.13 & 3.55 & & \\
\hline & 3 & 2.62 & 2.39 & & \\
\hline & 4 & 3.14 & 3.50 & & \\
\hline & 5 & 2.52 & 3.07 & & \\
\hline & 6 & 4.46 & 4.08 & & \\
\hline & Mean & 3.23 & 3.64 & & \\
\hline & 7 & & & 2.34 & 3.79 \\
\hline & 8 & & & 3.50 & 5.20 \\
\hline & 9 & & & 3.05 & 4.61 \\
\hline & 10 & & & 2.33 & 3.91 \\
\hline & 11 & & & 2.78 & 4.54 \\
\hline & 12 & & & 3.33 & 4.90 \\
\hline & Mean & & & 2.87 & 4.52 \\
\hline icc & 0.026 & & & & \\
\hline $\mathrm{P}$ & 0.23 & & & & \\
\hline
\end{tabular}

GDS: Geriatric Depression Scale, icc: Inter-cluster correlation, P: p-value of cluster adjusted Chi-squared test, SD: standard deviation

\section{Quality of life}


Quality of life measured according to the EQ5D 5L level between the intervention and control arm clusters was not significantly different ( $P=0.076$, icc 0.02 , cluster-adjusted t-test). (Table 11)

Table $11 \mathrm{EQ} 5 \mathrm{D} 5 \mathrm{~L}$ by clusters in intervention and control arms

\begin{tabular}{|c|c|c|c|c|c|}
\hline & \multirow[t]{2}{*}{ Clusters } & \multicolumn{2}{|c|}{ Intervention arm } & \multicolumn{2}{|c|}{ Control arm } \\
\hline & & Mean & SD & Mean & SD \\
\hline & 1 & 0.79 & 0.29 & & \\
\hline & 2 & 0.82 & 0.23 & & \\
\hline & 3 & 0.84 & 0.19 & & \\
\hline & 4 & 0.81 & 0.27 & & \\
\hline & 5 & 0.77 & 0.28 & & \\
\hline & 6 & 0.75 & 0.28 & & \\
\hline & Mean & 0.80 & 0.026 & & \\
\hline & 7 & & & 0.79 & 0.26 \\
\hline & 8 & & & 0.78 & 0.28 \\
\hline & 9 & & & 0.82 & 0.25 \\
\hline & 10 & & & 0.83 & 0.22 \\
\hline & 11 & & & 0.87 & 0.22 \\
\hline & 12 & & & 0.75 & 0.31 \\
\hline Mean & & & & 0.80 & 0.26 \\
\hline icc & 0.0190 & & & & \\
\hline$P$ & 0.76 & & & & \\
\hline
\end{tabular}

EQ5D5L: European Quality of Life scale, icc: Inter-cluster correlation, Cl: 95\% confidence interval: P: p-value of cluster adjusted Chi-square, SD: standard variation

\section{Intervention Cost of CIIC}

The Number Needed to Treat (NNT) for reducing the family burden was 9.5 (Table 4, Figure 3) and the NNT for care prevention was 20 (Table 7, Figure 3). We calculated the cost of each component of CIIC intervention in terms of capital cost, operational labor cost and operational material cost. The cost to provide care prevention intervention is 149 THB per six months per person. In relation to reducing the family burden, the cost of providing family caregiver capacity-building and standby respite care is 669 THB per six months per person and 294 THB per six months per person without respite care. (Figure 3) 


\section{Discussion}

Populations around the world are ageing faster. In 2021, at the start of global healthy ageing decade, there were more than 1 billion people aged over 60 years around the world, with the majority living in low- and middle-income countries, where health and social care are yet to be fully-inclusive of the ageing population. (31) Furthermore, multiple barriers confront the full participation of these older people in society. Communityintegrated innovative models, which would secure access to health and social care for the older persons, providing the basic resources necessary for a life of meaning and dignity, are urgently required. The results of this cluster randomized controlled trial, carried out in the setting of Chiang Mai, northern Thailand, have shown that CIIC is an intervention model which may be effective in preventing long-term care need (Table 7) and in reducing the burden on family caregivers (Table 4) amidst the ongoing COVID-19 pandemic. It can enhance the capacity of the caring families, which constitutes the core of the existing traditional long-term care model. ( Figure 3) Moreover, it may serve to prevent the long-term care need through communityintegrated health promotion by preserving the functional capacity of the older persons. (Figure 3 )

Health and social care in the community is the dream for many developing countries around the world. The increasing role of municipalities to be involved in health and long-term care, within a decentralized approach, requires a community-integrated model which is effective and sustainable in terms of the municipality setting and funding.(32) CIIC is a new concept and a new service model based on preventive strategy implemented through community integration. (Figure 2) The type of service is intermediary. It is a combination of formal care and informal care in a particular community, subdistrict, district, city or country. It is an integration of primary health care, social care and health promotion in the community setting. (Figure 2) Locating CIIC services in a neighborhood environment is designed to raise local attention and people's awareness, familiarity, and responsibility within the social network of the local community.

The results of this study saw a significant reduction of the caregiver burden in the intervention arm. (Table 4) CIIC provided technical guidance and advice for caregiving depending on the needs of the families with dependent older adults. CIIC nurses and volunteers visited participants' houses and trained them in caregiving techniques. CIIC services to enhance the family caregiver's capacity were provided to home-bound patients in integration with the primary health care centre. Caregivers could acquire formal information through the CIIC team, especially from the nurse and training of the tasks which require manual dexterity and technique to carry them out, such as hygiene tasks (bed bathing), transfer and mobility. As a result, the family caregiver's burden decreased in the intervention arm in comparison with the routine care in Thailand. Improving the caregiver's skills, together with providing mental support via access to short-term respite care services in the local community when feeling burdened or burnout could relieve the burden on those informal caregivers. There was less respite home admission, whereas home visits and community outreach became the main service. The determining factor was the safety concern for facility-based care during the COVID-19 pandemic. Evidence in other settings also reported the increasing utilization of home-based and family-based services during the COVID-19 pandemic.(33)

Moreover, caregiver burden measurement of the family caregivers can be applied as a screening tool to assess long-term care needs, complementing the routine dependency assessment of care recipients using ADL scoring. $(11,14)$ In many developing countries, studies increasingly have reported the long-term care need 
among older persons. $(34,35)$ National programs have started to screen for frailty among the older persons with several kinds of frailty screening tools. In a setting where long-term care is mainly provided through unpaid, informal care by families, the decision to grant formal long-term care service will require assessment of the family caregiver burden, in addition to the older person's care need. $(14,36)$ Moreover, the assessment should be applicable in the community setting as understandable evidence for stakeholders.

In this intervention study, we introduced three steps as part of a prevention strategy employing CIIC services. The first step was a co-creation of community group exercise for prevention of long-term care need. Participation in such community exercises kept the seniors active, connected and independent, with the potential outcome that long-term care need will be reduced [22]. Home exercise, which was designed as an alternative to group exercise, became a major breakthrough as an intervention to sustain care prevention during the COVID-19 pandemic.

Functional training exercises have been reported as effective ways to prevent frailty.(37) Effectiveness in this study related to the delivery model that we used to introduce care prevention exercise to the Thai community. Community volunteers were trained to be the leaders for group exercise activities, with training lasting four days. The schedule, place and time were determined according to the community stakeholder's meeting and older persons' decision. The researcher introduced the techniques and required devices, the protocol for safety, and the alternative home exercise option. Municipality officers coordinated these events. Recently, the World Health Organization Centre for Health Development referred to such activities as community-based social innovation (CBSI) for healthy ageing.(38) CBSI in Thailand is usually part of a state-driven model. In the CIIC model, the technique and devices were offered from the research, but community members decided everything else. Practically it promoted autonomy, civic participation, and dignity of the older persons.

(39) CIIC applied community empowerment for launching care prevention exercise to prevent frailty. Consequently, the burden on the caregivers decreased. (Table 3,4,6 and7)

Second, when there is little care need, the older people can enjoy staying at home with their families, thereby improving their chances of "ageing in place". [4] Families were assisted through training, direct help and respite service provided by the CIIC [9]. When family caregivers are faced with extreme burden and burnout, the older persons will be able to access a formal care service in the community. Again, the hope is that it can prevent unnecessary long-term care costs. CIIC offers an affordable model integrating formal services and informal care by families.(Figure 3) It may be applicable in many resource-limited countries helping to establish a fully-structured, formal long-term care service.

Third, when older people remain active and healthy, their health care needs may be reduced thereby avoiding unnecessary hospital admission and reducing health care costs. Further analysis of CIIC for cost and benefits will be required. Despite the higher stress due to the COVID19 pandemic, older residents who had CIIC in their communities were less likely to get frail, compared to the residents in the control sites. Prevention of care need also reduced the burden on family caregivers. ( Table 4and 7) Therefore, this CIIC model may promote active ageing in place, and prevent the long-term care and health costs.

In addition, the screening of activities of daily living and of common non-communicable diseases was able to identify unmet needs earlier and so prevent age-related morbidity and disability. Frailty screening is

Page 23/31 
haphazardly carried out by primary health care centres. CIIC introduced a dedicated facility and staff for screening frailty which is internationally recommended. $(40,41)$ Therefore, in the long-term, the interaction of CIIC services and primary health care services could be harmonized, leading to better integration and represent a step to realizing universal access to long-term health care in Thailand.

\section{Lesson in implementation}

A range of lessons were learnt in developing and implementing CIIC. Researchers sought shared value with partners such as the municipality authority, primary health care providers, and the Ministry of Public Health. Initial advocacy applied a shared narrative to explain why integrated care matters. A persuasive vision was described to stakeholders about what CIIC could achieve. It is because we established shared leadership among the study site municipality administration, the public health authority and the researchers, that communities could find the venue, time, professionals, and volunteers, and mobilize resources to develop a community respite facility and coordination of community-based care prevention activities for older persons. This represented a new understanding and a new way of working. The services of CIIC, such as care prevention, empowerment of family caregivers, and community respite, were well-identified, and the older community residents could see the real benefits from the integrated services.

Moreover, community-integrated care was developed both from the bottom up, mobilizing community resources, as well as from the top down, triggering interest, commitment and involvement of the public health authority. CIIC could support and empower caregivers and older people to take more control over their health and wellbeing. Formal and informal sectors were integrated effectively in an innovation model, to maintain the intrinsic capacity of the older persons and to reduce family caregivers burden leading to a specific objective of a healthy ageing community, with realistic costs of integrated care. If the model can be applied and sustained in the community, routine identification of frailty and risk stratification can be readily applied.

However, the COVID-19 pandemic delayed the intervention and reduced the participation. After six months of CIIC services, there was a significantly lower caregivers' burden. The COVID-19 pandemic impeded the implementation and might have reduced the effect size. (Table 5,8 and11) Despite such a situation and comparing to active control, CIIC interventions saw effectiveness in enhancing family care capacity and preventing long term care, with low cost in a six months implementation period.

Facing the increasing burden of non-communicable diseases and age-related morbidity, Thai communities are increasingly in need of community-integrated care models for older persons that can link existing health systems and reduce the burden upon caring families. This need is common to many countries in the Association of Southeast Asian Nations (ASEAN) and around the world. The role of informal caregiving is more emphasized in high-income countries recently whereas there is very little formal caregiving capacity in low-income countries. Since ASEAN and many Asian countries share similar traditional family-based, longterm care systems, the protocol, implementation experiences, results and products from CIIC may provide evidence-based policy options for other countries wishing to adopt similar community-integrated care models for older people. 
The trial was carefully designed to have enough power to see intervention effectiveness compared to the active control. This CIIC trial implementation was impacted by the COVID-19 pandemic, as with many trials across the world. However, we retained a large sample at randomization and analysis. Our backward calculation revealed that the power of the sample at analysis was 0.79 which was adequate for the effect size of the finding relating to the primary outcome of the caregiver burden. Within a short implementation time of six months, interrupted by the pandemic-related situations, we could see the effectiveness of the community-integrated intermediary model in reducing the caregivers' burden (Table 4) and preventing functional decline of the older people. (Table7) Yet, the effectiveness of CIIC as reported in this report may only represent the tip of iceberg. Sustainability and maintenance of implementation is required to see the full effectiveness and provide stronger evidence for the ageing world. This intervention study of a comprehensive model, well-integrated into the community, is the first to have been carried out in Thailand and represents an initiative for the rest of Asia and the world. Opportunities for scaling up CIIC in future research remain.

\section{Conclusion}

This study had shown the CIIC model as an effective and potential step to the realization of universal health and long-term care coverage being inclusive of the ageing population in Thailand. The evidence and lessons learnt in this study are expected to be beneficial in order to scale up CIIC across Thailand, other similar countries in Asia and around the world.

\section{List Of Abbreviations}

$\mathrm{ADL}$

activities of daily living

ASEAN

Association of Southeast Asian Nations

CBI

Caregiver Burden Inventory

CIIC

community-integrated intermediary care

GDS

Geriatric Depression Scale

MANOVA

multivariate analysis of variance

QOL

quality of life

SD

standard deviation

\section{Declarations}


Ethics approval and consent to participate This research project was approved by the Research Ethics Review Committee of the World Health Organization (protocol no. ERC.0003064) and Boromrajonani College of Nursing, Lampang, Thailand Ethics Review Committee (approval no. E2562/005). The study is registered in the Thailand Clinical Trial Registry, with trial registration number TCTR20190412004. Written informed consent to participate was obtained from all subjects involved in the study.

Consent for publication Written informed consent for publication of their clinical details was obtained from the study participants.

Availability of data and materials The datasets used and analysed during the current study are available from the corresponding author on reasonable request.

Competing interests The authors declare that they have no competing interests.

Funding This research was supported by the World Health Organization Centre for Health Development (WHO Kobe Centre - WKC: K18020). The funders had no role in the design of the study; in the collection, analyses or interpretation of data; in the writing of the manuscript; or in the decision to publish the results.

Authors' contributions MNA idealized and designed the CIIC model. M.N.A., M.Y. and S.M. designed interventions. Y.K. developed the care prevention exercise plan. M.Y., and T.N.N.A designed family capacity building intervention. M.N.A, M.Y and S.M. developed randomization and follow up plan. S.S., A.K., S.C developed implementation plan. Every author made substantial contributions to data collection, analysis and writing the manuscript in several versions. S.M, S.S, A.K., S.C commented cost and collaboration lesson. T.N.N.A edited discussion related to family care capacity building. M.N.A drafted the first version manuscript and finalized the manuscript after everyone commented on it. All authors have read and approved the final manuscript.

Acknowledgements Richard Lawrence Mann is acknowledged for refining English. The mayor of Maehia municipality and the mayor's team are acknowledged for their contribution to establishing the CIIC facility and enabling community empowerment. The mayor of Suthep municipality and the mayor's team are acknowledged for their cooperation. The Faculty of Science and Technology, Chiang Mai Rajabhat University, Chiang Mai, Thailand, and the Japan International Cooperation Agency are acknowledged for their contribution to and cooperation in the CIIC study.

\section{References}

1. NSO. Demography Population and Housing Branch: Size and structure of the population by age and sex 2021: National Statistical Office Thailand; 2021 [Available from:

http://statbbi.nso.go.th/staticreport/page/sector/en/01.aspx.

2. Abbafati C, Abbas KM, Abbasi M, Abbasifard M, Abbasi-Kangevari M, Abbastabar $\mathrm{H}$, et al. Global burden of 369 diseases and injuries in 204 countries and territories, 1990-2019: a systematic analysis for the Global Burden of Disease Study 2019. Lancet. 2020;396(10258):1204-22. 
3. Suriyanrattakorn S, Chang C-L. Long-term care (LTC) policy in Thailand on the homebound and bedridden elderly happiness. Health Policy OPEN. 2021;2:100026.

4. Barber SL, van Gool K, Wise S, Wood M, Or Z, Penneau A, et al. Pricing long-term care for older persons. Geneva: World Health Organization; 2021.

5. Chanprasert P. Long-term Care Policy and Implementation in Thailand'. In: O K, Saito Y, editors. Coping with Rapid Population Ageing in Asia: Discussions on Long-term Care Policy and Cross-border Circulation of Care Workers. Jakarta: Economic Research Institute for ASEAN and East Asia; 2021. pp. 36-44.

6. Knodel J, Kespichayawattana J, Wiwatwanich S, Saengtienchai C. The future of family support for Thai elderly: Views of the populace. Journal of Population and Social Studies. 2013;Volume 21 Number 2 , January 2013: 110-132.

7. Aung MN, Moolphate S, Aung TNN, Katonyoo C, Khamchai S, Wannakrairot P. The social network index and its relation to later-life depression among the elderly aged $\geq 80$ years in Northern Thailand. Clin Interv Aging. 2016;11:1067.

8. Knodel J, Nguyen MD. Grandparents and grandchildren: care and support in Myanmar, Thailand and Vietnam. Ageing Soc. 2015;35(09):1960-88.

9. Mudrazija S. Work-Related Opportunity Costs Of Providing Unpaid Family Care In. 2013 And 2050. Health Aff (Millwood). 2019;38(6):1003-10.

10. Suzuki Y, Honjo K. The association between informal caregiving and poor self-rated health among evermarried women in Japan: A nationally representative survey. J Epidemiol. 2020.

11. Aung TNN, Aung MN, Moolphate S, Koyanagi Y, Supakankunti S, Yuasa M. Caregiver Burden and Associated Factors for the Respite Care Needs among the Family Caregivers of Community Dwelling Senior Citizens in Chiang Mai, Northern Thailand. International Journal of Environmental Research Public Health. 2021;18(11):5873.

12. Yon Y, Mikton CR, Gassoumis ZD, Wilber KH. Elder abuse prevalence in community settings: a systematic review and meta-analysis. Lancet Glob Health. 2017;5(2):e147-e56.

13. Kodate N, Timonen V. Bringing the family in through the back door: The stealthy expansion of family care in asian and european long-term care policy. J Cross-Cult Gerontol. 2017;32(3):291-301.

14. Aung TNN, Aung MN, Moolphate S, Koyanagi Y, Ichikawa M, Supakankunti S, et al. Estimating Service Demand for Intermediary Care at a Community Integrated Intermediary Care Center among Family Caregivers of Older Adults Residing in Chiang Mai, Northern Thailand. International Journal of Environmental Research Public Health. 2021;18(11):6087.

15. Vecchio N, Fitzgerald JA, Radford K, Kurrle S. Respite service use among caregivers of older people: comparative analysis of family dementia caregivers with musculoskeletal and circulatory system disorder caregivers. Aging Ment Health. 2018;22(1):92-9.

16. Greiner C, Tamdee D, Okamoto N, Tamdee P, Isowa T, Booonchiang W, et al. Comparison survey on family caregivers of elderly people in Thailand and Japan. Innovation in Aging. 2017;1(suppl_1):601-.

17. Thinuan P, Siviroj P, Lerttrakarnnon P, Lorga T. Prevalence and Potential Predictors of Frailty among Community-Dwelling Older Persons in Northern Thailand: A Cross-Sectional Study. International Journal 
of Environmental Research and Public Health. 2020;17(11).

18. Theou O, Rockwood K. Frailty in Aging: Biological, Clinical and Social Implications. Karger Medical and Scientific Publishers; 2015.

19. Saito M, Aida J, Kondo N, Saito J, Kato H, Ota Y, et al. Reduced long-term care cost by social participation among older Japanese adults: a prospective follow-up study in JAGES. Bmj Open. 2019;9(3).

20. Aung MN, Koyanagi Y, Ueno S, Tiraphat S, Yuasa M. Age-friendly environment and community-based social innovation in Japan: A mixed-method study. Gerontologist. 2021.

21. Enhancing family based long term care with a model of community integrated intermediary care (CIIC) service for Thai older adults in Chiang Mai, Thailand: cluster randomized controlled trial [Internet]. World Health Organization, International Clinical Trials Registry Platform (ICTRP). 2019. Available from: https://trialsearch.who.int/Trial2.aspx?TrialID=TCTR20190412004.

22. Eldridge S, Kerry S. A practical guide to cluster randomised trials in health services research. John Wiley \& Sons; 2012.

23. Novak M, Guest C. Application of a multidimensional caregiver burden inventory. Gerontologist. 1989;29(6):798-803.

24. Valer DB, Aires M, Fengler FL, Paskulin LMG. Adaptation and validation of the caregiver burden inventory for use with caregivers of elderly individuals. Revista Latino-Americana de Enfermagem. 2015;23(1):130-8.

25. Barthel Activities of Daily Living (ADL). Index. Occasional paper (Royal College of General Practitioners). 1993(59):24-.

26. Department of Medical service MoPh. THAILAND. Manual of assessment tool for ederly people 2014.

27. Wolfs CA, Dirksen CD, Kessels A, Willems DC, Verhey FR, Severens JL. Performance of the EQ-5D and the EQ-5D+C in elderly patients with cognitive impairments. Health Quality of Life Outcomes. 2007;5(1):33.

28. EuroQolGroup T. EuroQol-a new facility for the measurement of health-related quality of life. Health Policy. 1990;16(3):199-208.

29. Randomized controlled trial to evaluate a model of community integrated intermediary care (CIIC) services for older adults in Thailand [Internet]. World Health Organization, Centre for Health Development. 2018. Available from: https://extranet.who.int/kobe_centre/en/project-details/asean_thailand.

30. Aung MN, Yuasa M, Koyanagi Y, Aung TNN, Moolphate S, Matsumoto H, et al. Sustainable health promotion for the seniors during COVID-19 outbreak: a lesson from Tokyo. J Infect Dev Ctries. 2020;14(4):328-31.

31. UN decade of healthay ageing 2021-2030: World Health Organization; 2021 [Available from: https://www.who.int/initiatives/decade-of-healthy-ageing.

32. Kim H, Kwon S. A decade of public long-term care insurance in South Korea: Policy lessons for aging countries. Health Policy. 2021;125(1):22-6.

33. Ito T, Hirata-Mogi S, Watanabe T, Sugiyama T, Jin X, Kobayashi S, et al. Change of Use in Community Services among Disabled Older Adults during COVID-19 in Japan. Int J Environ Res Public Health. 2021;18(3):1148. 
34. Win HH, Nyunt TW, Lwin KT, Zin PE, Nozaki I, Bo TZ, et al. Cohort profile: healthy and active ageing in Myanmar (JAGES in Myanmar 2018): a prospective population-based cohort study of the long-term care risks and health status of older adults in Myanmar. Bmj Open. 2020;10(10):e042877.

35. Thanyawinichkul P, Aung MN, Moolphate S, Katonyoo C, Chawapong W, Sennun P, et al. Dependency, Disability, Depression and Health Behaviors of the Oldest of the Old Community Residents: A Community Survey in Chiang Mai, Thailand. Journal of Public Health in Developing Countries. 2016;2(2):183-98.

36. Rodrigo-Baños V, Moral-Pairada MD, González-de Paz L. A Comprehensive Assessment of Informal Caregivers of Patients in a Primary Healthcare Home-Care Program. Int J Environ Res Public Health. 2021;18(21).

37. Apóstolo J, Cooke R, Bobrowicz-Campos E, Santana S, Marcucci M, Cano A, et al. Effectiveness of interventions to prevent pre-frailty and frailty progression in older adults: a systematic review. JBI Database System Rev Implement Rep. 2018;16(1):140-232.

38. Ghiga I, Pitchforth E, Lepetit L, Miani C, Ali G-C, Meads C. The effectiveness of community-based social innovations for healthy ageing in middle- and high-income countries: a systematic review. J Health Serv Res Policy. 2020;25(3):202-10.

39. Aung TNN, Aung MN, Moolphate S, Koyanagi Y, Mulati N, Supakankunti S, et al. Thai Older People's Willingness (Intention) to Participate in a Care Prevention, Community Group Exercise Program: An Assessment before Implementing an Intervention Trial in Chiang Mai, Northern Thailand. International Journal of Environmental Research Public Health. 2021;18(8):4044.

40. Ofori-Asenso R, Chin KL, Mazidi M, Zomer E, llomaki J, Zullo AR, et al. Global Incidence of Frailty and Prefrailty Among Community-Dwelling Older Adults: A Systematic Review and Meta-analysis. JAMA Netw Open. 2019;2(8):e198398-e.

41. Dent E, Morley JE, Cruz-Jentoft AJ, Woodhouse L, Rodríguez-Mañas L, Fried LP, et al. Physical Frailty: ICFSR International Clinical Practice Guidelines for Identification and Management. J Nutr Health Aging. 2019;23(9):771-87.

\section{Figures}




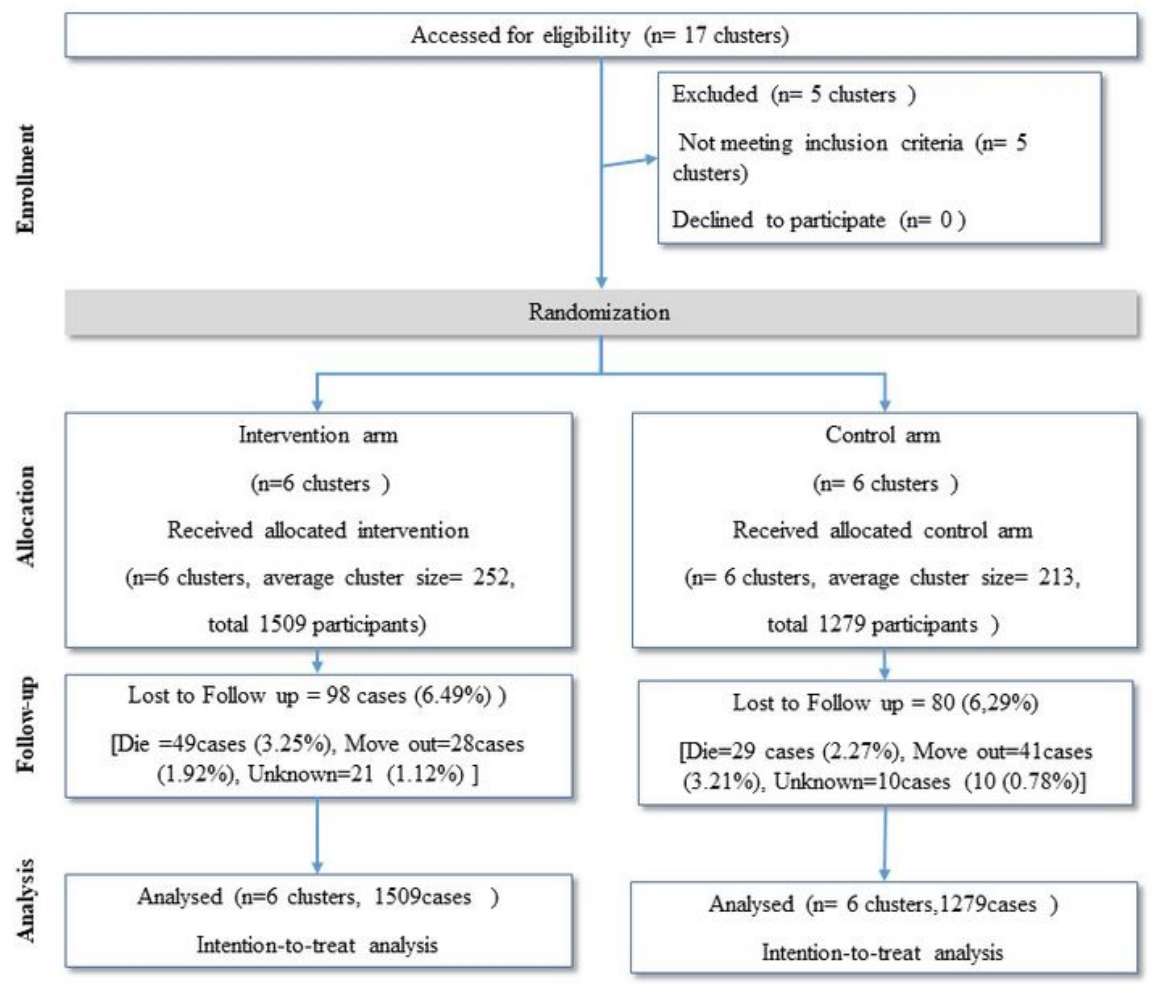

\section{Figure 1}

CONSORT diagram in the community integrated intermediary care model for long term care, Thailand

CIIC model

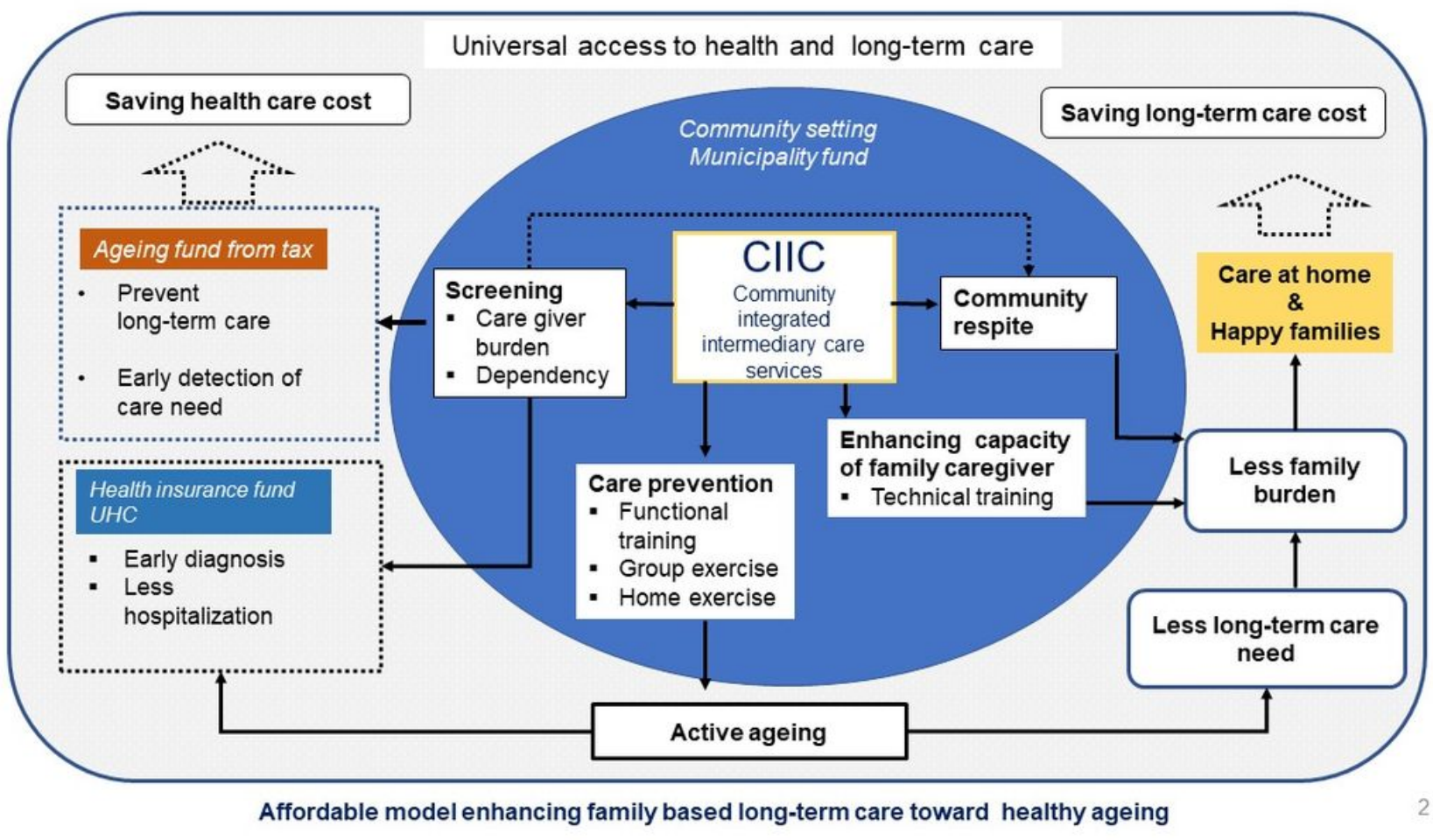


Figure 2

Service and contributions of community integrated intermediary care (CIIC) model explaining intervention components.

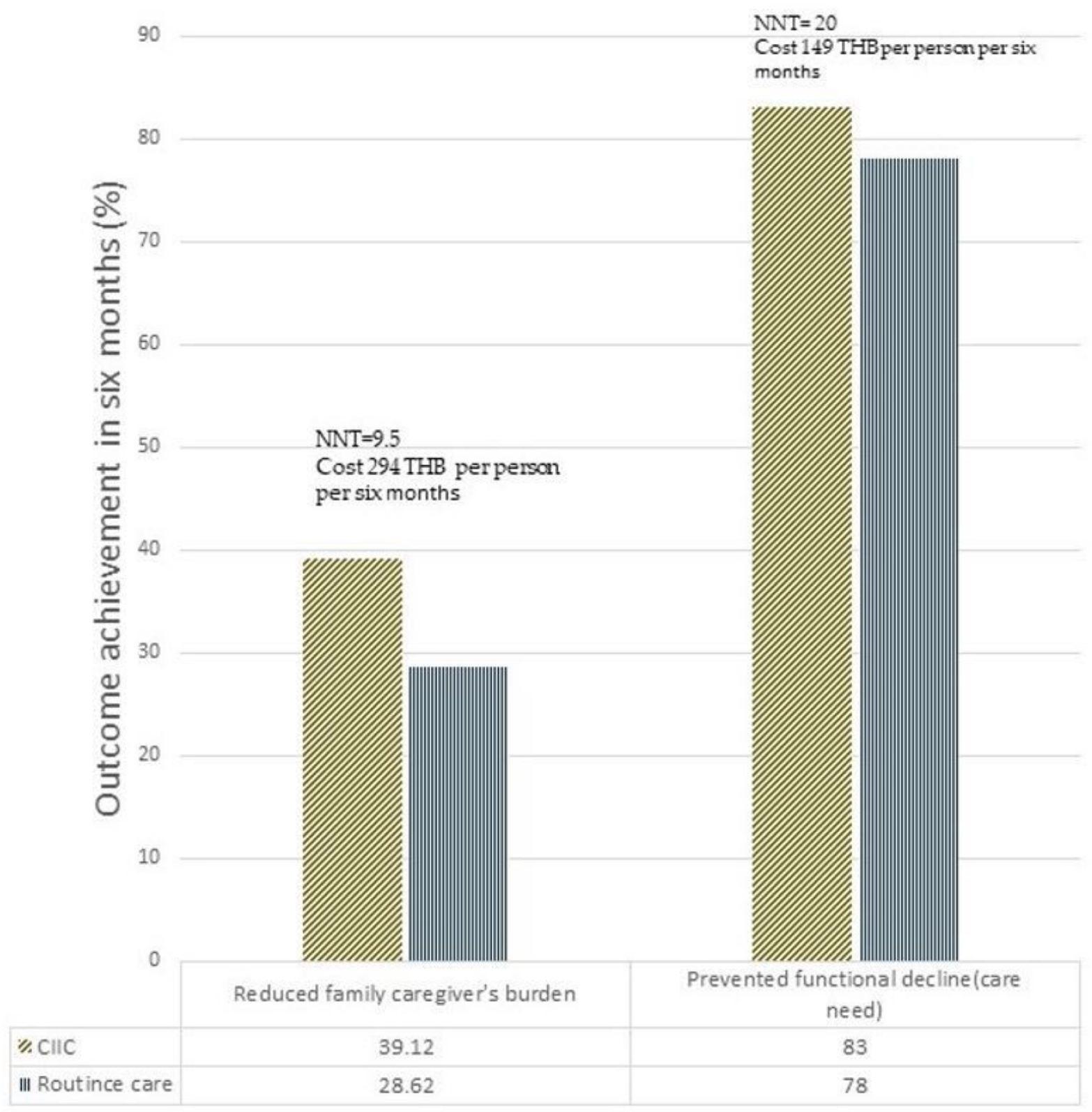

\section{Figure 3}

Effectiveness in six months and cost of enhancing family care capacity and long-term care prevention interventions components in community-integrated intermediary care CIIC trial during COVID-19 pandemic, Thailand 2021 ( $n=2788)$, THB Thai Baht ( Tentatively 1 THB=0.03 US Dollar as of current exchange rate) 